\title{
Cocaine Increases Mortality and Cardiac Mass in a Murine Transgenic Model of Acquired Immune Deficiency Syndrome
}

\author{
Roy L. Sutliff, Chad Haase, Rodney Russ, Brian D. Hoit, Randal Morris, \\ Andrew B. Norman, and William Lewis \\ Department of Experimental Pathology and Laboratory Medicine (RLS, CH, RR, WL), Emory University, Atlanta, \\ Georgia; and Department of Medicine (BDH), Case Western Reserve University, Cleveland, and Departments of Cell \\ Biology (RM) and Psychiatry (ABN), University of Cincinnati College of Medicine, Cincinnati, Ohio
}

\begin{abstract}
SUMMARY: Cardiac dysfunction in AIDS is an important problem. Cocaine is an epidemic associated with sudden death, cardiac dysfunction, and congestive heart failure. Cocaine use and HIV infection frequently coexist in the same patient, yet the combined impact of both is poorly understood. The present study uses cocaine treatment of an established murine AIDS transgenic model (NL4-3s gag/pol; TG) to define the combined effects of AIDS and cocaine on cardiac pathophysiology. To determine the effects of cocaine and HIV-1 proteins on mortality, wild-type and NL4-3 $\Delta$ gag/pol mice received saline or cocaine via continuous infusion by Alzet osmotic pumps for 28 days (chronic). Acute cocaine administration (10 days; $40 \mathrm{mg} / \mathrm{kg} / \mathrm{day}$ ) was used to study the nonlethal effects of cocaine in TGs. Echocardiograms and single time point electrocardiograms were performed at the termination of each experiment. Hearts were removed and examined histopathologically. Chronic cocaine treatment $(80$ $\mathrm{mg} / \mathrm{kg} / \mathrm{day} ; 28$ days) markedly decreased median survival in both wild-type and TG; however, TG survival was significantly more decreased. In acute studies, TG echocardiographic changes included increased left ventricular mass and increased left ventricular fractional shortening compared with all cohorts. Electrocardiographic changes were absent among the groups. Histopathologically, perivascular fibrosis and interstitial fibrosis were evident in cocaine-treated TG. Data suggest that additive cardiac insults (from AIDS and cocaine) result in combined deleterious effects. (Lab Invest 2003, 83:983-989).
\end{abstract}

$C$ ocaine may contribute to the development of cardiomyopathy (CM) in AIDS as a toxin to myocardial cells or through indirect mechanisms based on cocaine's vascular effects and secondary myocardial changes. Accordingly, cardiac effects of cocaine may be a comorbid condition in AIDS CM and vice versa.

Cocaine is rapidly cleared and distributed to heart, brain, spinal cord, and other tissues (Inaba et al, 1978; Som et al, 1994; Volkow et al, 1992). Excellent data regarding $\mathrm{CM}$ in chronic cocaine administration in humans comes from heart transplantation studies (Karch and Billingham, 1988). Morphologic features in native hearts from chronic cocaine abusers who underwent heart transplantation differed from those seen in non-cocaine abusers.

The purpose of our experiments was to address the individual, additive, or synergistic deleterious effects of cocaine and AIDS on the development of cardiac structural and functional abnormalities. We used an

DOI: 10.1097/01.LAB.0000075555.93242.F2

Received February 27, 2003.

This work was supported by Grants 5R01HL059798 and 5R01HL065167 (to WL) and AHA003279 (to RLS).

Address reprint requests to: Dr. W. Lewis, Professor of Pathology, Emory University, 1639 Pierce Drive, Room 7117 WMRB, Atlanta, Georgia 30322. E-mail:wlewis@emory.edu established AIDS transgenic model [NL4-3山 gag/pol (TG); reviewed in Klotman and Notkins, 1996] that we have used in the past to study CM in AIDS (Lewis et al, 2001a, 2000). Mice received continuous cocaine infusion via miniosmotic pumps for 10 days (acute) and 28 days (chronic). Treatment with high-dose cocaine decreased survival in chronic studies. Cocaine-treated TGs develop pathologic lesions including vascular and interstitial fibrosis. Functionally, left ventricular (LV) mass and LV fractional shortening increased in TGs treated with cocaine. Together, data indicate that cocaine has significant deleterious effects on the cardiac and vascular system in AIDS and may worsen cardiac disease in this subset of substance abusers.

\section{Results}

\section{Survival from Procedure and Kaplan-Meier Analysis of Wild-Type (WT) and TG Treated with Cocaine}

The operative mortality from osmotic pump placement was below 4\% in both TG and WT. Anesthetic overdose seemed to be responsible for operative deaths. To establish the combined effects of cocaine and AIDS on survival, Kaplan-Meier survival curves were generated for WT and AIDS TG cohorts treated with saline or cocaine (40 or $80 \mathrm{mg} / \mathrm{kg} /$ day). AIDS TG (cocaine $80 \mathrm{mg} / \mathrm{kg} /$ day) exhibited significantly reduced median survival time compared with either 
cocaine-treated WT controls or untreated AIDS TG (Fig. 1). The median survival time of the AIDS TG cohort was 458 hours. Continuous infusion of cocaine ( $80 \mathrm{mg} / \mathrm{kg} /$ day) reduced the median survival time of WT mice to 291 hours, whereas survival time of AIDS TG was significantly lower (49.5 hours; $p<0.05)$. Lower doses of cocaine (40 mg/kg/day) did not affect the survival of the AIDS TG compared with either WT or TG saline controls (Fig. 1).

\section{Echocardiography of WT and TG Treated with Cocaine}

M-mode echocardiography was used to determine the impact of cocaine on cardiac function and mass of WT and TG mice. Heart rate was similar in WT (with or without cocaine treatment) and in untreated TG (Fig. 2A). However, a slight but significant $(p<0.05$ ) increase in heart rate was observed in the transgene cohort that received cocaine. Administration of cocaine to TG mice resulted in a significant increase in left ventricular end diastolic dimensions (Fig. 2B). LV mass (normalized to body weight; $\mathrm{mg} / \mathrm{gm}$ ) was strikingly increased in hearts from the TG cohort that received cocaine (Fig. $2 \mathrm{C}$ ). Cocaine treatment of $\mathrm{TG}$ increased left ventricular fractional shortening $(p<$ 0.05; Fig. 2D). Relative wall thickness was unchanged among the cohorts (Fig. 2E).

\section{Electrocardiography of WT and TG Treated with Cocaine}

Development of cardiac arrhythmias from cocaine administration was evaluated in AIDS TGs and WT littermates using the AnonyMOUSE system described below. A significant increase in the QRS duration and QT interval were found in untreated TGs (Fig. 3). Curiously, cocaine (40 mg/kg/day; 10 days) treatment of TGs reduced this QRS duration and QT interval. Cocaine administration did not affect cardiac rhythm of WT.

\section{Histologic Features of the Hearts from WT and TG Treated with Cocaine}

Structural changes were defined histopathologically in AIDS TGs and WT controls with cocaine $(40 \mathrm{mg} / \mathrm{kg} /$

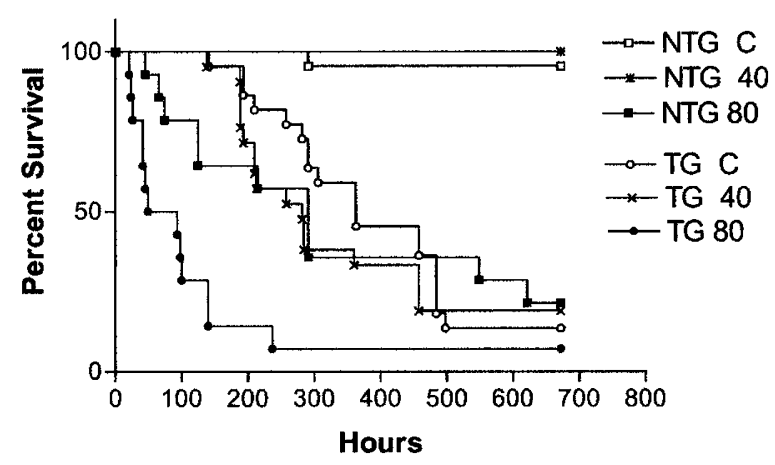

Figure 1.

Kaplan-Maier survival curves of WT and TG mice as a function of cocaine dose (40 and $80 \mathrm{mg} / \mathrm{kg} / \mathrm{day}$ ). Mice were implanted with miniosmotic pumps for continuous infusion of cocaine or saline for 28 days. Cocaine treatment was associated with premature death $(n=15-22)$. day) or saline treatment (described above). Figure 4 shows representative photomicrographs for the respective transgenic and WT cohorts with and without cocaine. Semiquantitative analysis of myocardial perivascular fibrosis, myocardial fibrosis, and myocardial necrosis are summarized in the histogram (Fig. 5). Analysis of vascular and perivascular zones from histopathologic sections revealed thickening in the vascular wall, which is most prominent in the TG cohort treated with cocaine $(40 \mathrm{mg} / \mathrm{kg} /$ day). Additionally, perivascular interstitial fibrosis was recognized. This fibrosis extended into the interstitium surrounding the perivascular cardiac myocytes (Fig. 4). Myocyte necrosis was not increased by cocaine.

\section{Discussion}

Both cocaine use and HIV-1 are associated with cardiac dysfunction (reviewed in Lewis, 2000). Using a transgenic AIDS model and an approach that resembled those recently used (Lewis, 2001a, 2001b; Lewis et al, 2001a; Raidel et al, 2002) by our group, this study addressed the contribution of HIV-1 (as expressed in a transgenic HIV-1 construct) and cocaine to the development of CM in AIDS and conversely the impact of AIDS on cocaine cardiotoxicity. Our results indicate that cocaine and HIV-1 together impact on LV mass, and the presence of both conditions impairs cardiac function and accelerates mortality compared with either parameter alone.

It was reasonable to expect that the severity of the respective clinical conditions would result in combined deleterious effects. Recently, we demonstrated in vivo that transgenic mice harboring HIV-1 constructs (Lewis et al, 2000, 2001b) or selected HIV-1 gene products (Raidel et al, 2002) exhibit cardiac dysfunction. It has been known for some time that cocaine acutely and chronically impacts cardiac dysfunction in humans (Isner et al, 1986; Karch and Billingham, 1988). Accordingly, cocaine treatment of TGs and WTs resulted in differential mortality in chronic treatment protocols. Cocaine ( $80 \mathrm{mg} / \mathrm{kg} / \mathrm{day})$ administration increased mortality in both AIDS TG and WT mice compared with saline controls; however, mortality was significantly accelerated in AIDS TG using that treatment protocol. These findings indicate that the HIV-1 transgene and cocaine acted cooperatively with respect to mortality.

Pathologic changes that are consistent with cardiac dysfunction were found in the hearts of TGs and WTs treated with cocaine for 10 days. Cardiomegaly in cocaine-treated TGs correlated with increased LV cavity size pathologically and correlated with the echocardiographic findings of increased LV mass. The pathologic changes resembled findings with cocaine treatment reported by others in which fibrosis was a prominent feature that contributes to cardiac dysfunction. Of note, patchy fibrosis is a feature of chronic cocaine administration in the human heart.

Our results clearly demonstrate cocaine-induced remodeling in both WT and TG mice. Remodeling encompasses a complex of molecular and cellular 

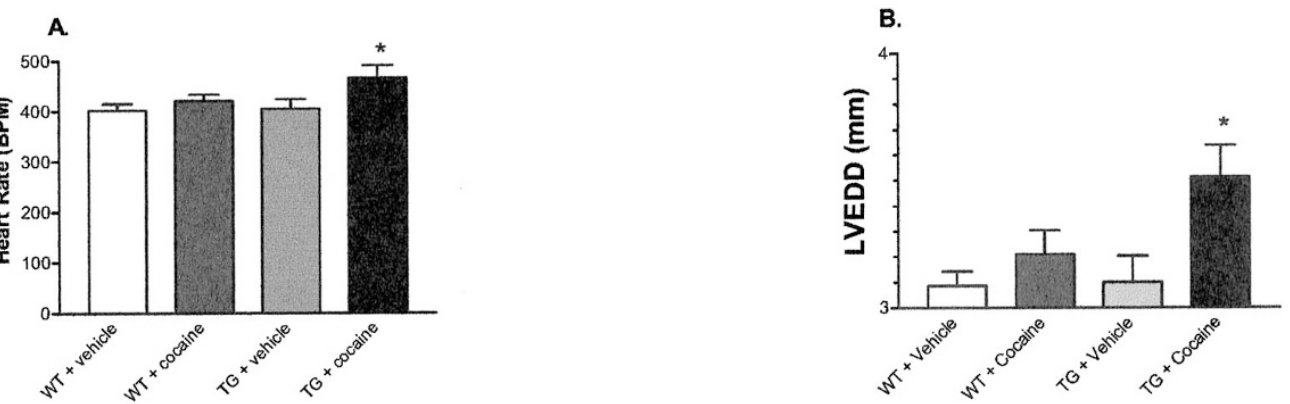

D.
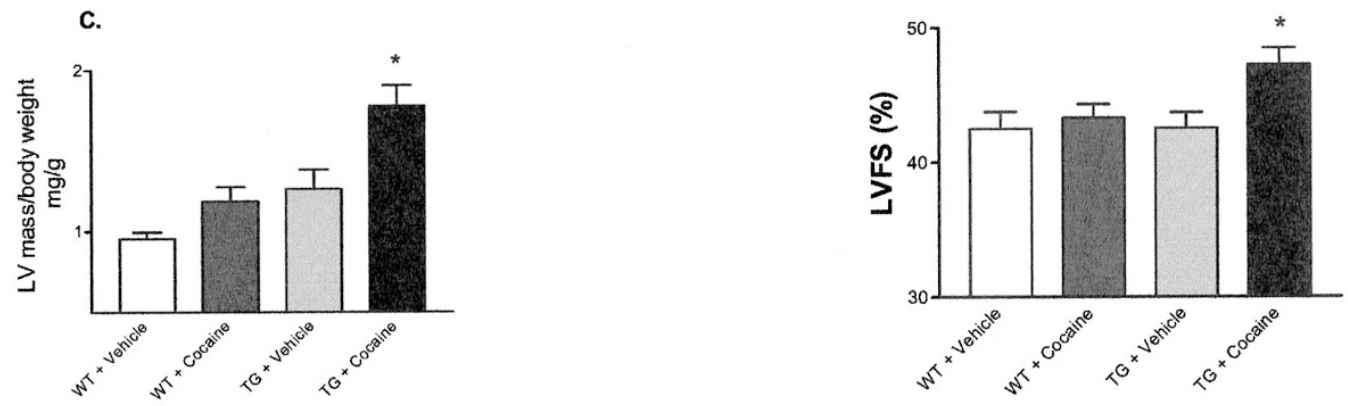

E.

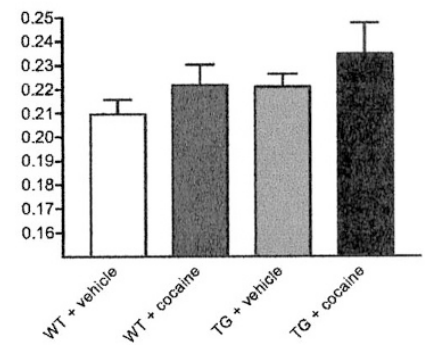

Figure 2.

Echocardiographic measurements of WT and TG mice treated with saline or cocaine (40 mg/kg/day). Mice were implanted with miniosmotic pumps for continuous infusion of cocaine or saline for 10 days and terminal echocardiography was performed. Parameters for heart rate (A); left ventricular end diastolic dimension ( $L V E D D$, B); LV mass normalized to body weight (C); left ventricular fractional shortening (LVFS, D); and posterior wall thickness (E) were calculated as described in "Materials and Methods." * $p<0.05$ compared with all other cohorts $(n=6-8)$.

events that lead to changes in the structure, function, and phenotype of the myocardium and is reflected in the increased LV mass and alterations in the quantity and composition of the extracellular matrix. Although increased activity of the sympathetic nervous system may account for cocaine-induced cardiac remodeling, the mechanisms underlying the more severe phenotype in TGs remain unclear. Data from our group and others suggests that HIV-1 proteins (ie, Tat) expressed in the heart can impact on cardiac function and/or cardiac cell death and this may in turn enhance remodeling responses to cocaine (Raidel et al, 2002; Twu et al, 2002).

Echocardiographic data from chronic treatment supported the pathophysiologic findings of CM using methods that we employed in the past (Lewis et al, $2000,2001 b)$. After 10 days of cocaine treatment (40 $\mathrm{mg} / \mathrm{kg} /$ day), data indicate that the combination of the $\mathrm{TG}$ and cocaine, but neither alone, results in cardiac enlargement. The posterior wall thickness was un- 

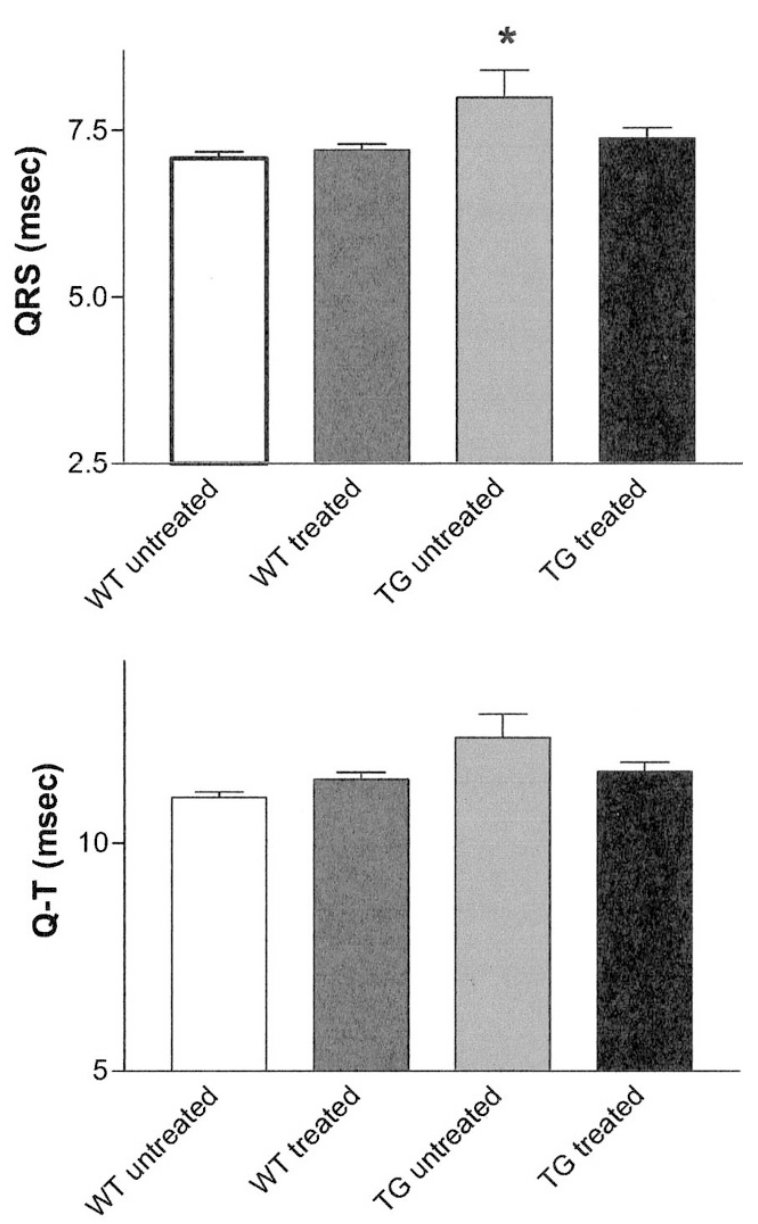

Figure 3.

Electrocardiographic measurement of cardiographic measurements of WT and TG mice treated with saline or cocaine $(40 \mathrm{mg} / \mathrm{kg} /$ day). Mice were implanted with miniosmotic pumps for continuous infusion of cocaine or saline for 10 days and electrocardiography was determined. QRS duration (A) and Q-T duration were measured $(n=6-8)$.

changed, suggesting that eccentric hypertrophy is present. Surprisingly, systolic performance was elevated after 10 days of cocaine treatment as demonstrated by the increased left ventricular fractional shortening. Heart rate, although elevated in TG mice treated with cocaine, is unlikely to explain entirely the increased LVFS, because these changes are small and in the range where the force-frequency relation is not that pronounced in anesthetized mice (Georgakopoulos and Kass, 2001).

Cardiac arrhythmias are often associated with cocaine abuse. Our data suggest that arrhythmias may not be present in this cocaine/AIDS cardiotoxicity model in which electrocardiograms (ECG) were performed for a short time. This maybe related to the relatively short period of time, 10 days, of cocaine treatment. Additionally, this lack of an effect on cardiac rhythm may be the result of the higher sympathetic drive that has been reported in the $\mathrm{FVB} / \mathrm{n}$ strain of mice (Shusterman et al, 2002). In this study, HR responses of $\mathrm{FVB} / \mathrm{n}$ mice to isoproterenol infusion were significantly lower than other strains of mice examined (Shusterman et al, 2002). The higher sym- pathetic drive in the FVB/n strain of mice may minimize the impact of cocaine on sympathetic responses such as heart rate. Furthermore, this is consistent with profound strain differences in heart rate responses to cocaine that have been previously reported (Ruth et al, 1988).

On a clinical basis, the role of cardiac HIV-1 infection in the pathogenesis of AIDS CM remains controversial, but infection of the myocytes or interstitial cells by HIV or simian immune deficiency virus, respectively, has been reported in both human samples and in nonhuman primate species infected in the laboratory. HIV-1 was isolated and cultivated from an endomyocardial biopsy specimen obtained from a patient with AIDS CM (Calabrese et al, 1987). Our group and others identified HIV-1 in human myocardial tissues or primate tissue (in which simian immune deficiency virus infection was detected) by in situ hybridization with radiolabeled riboprobes (Grody et al, 1990; Lipshultz et al, 1990; Shannon et al, 2000; Wu et al, 1990).

The impact of cocaine on the development of clinical AIDS CM remains unclear among the different populations at risk, but the importance of increasing numbers of HIV-infected patients and continued cocaine use cannot be ignored. In children, one group of investigators found progressive LV dilation in HIV-1infected pediatric patients irrespective of antiretroviral therapy (Lipshultz et al, 1992). Substance abuse was not addressed in that clinical study, and the TGs here were age-matched adults.

Early in the AIDS epidemic, myocarditis received attention as a pathophysiologic correlate to CM. Its pathogenetic role in AIDS CM remains controversial, despite a reported high prevalence in some studies and lower prevalence in others. No lesions of active myocarditis were present histopathologically in the heart samples from either the acute or chronic cocaine administration here. This suggests that the presence of inflammation (on a histopathologic basis) is not necessary for the development of features of cardiac pathologies.

In summary, this AIDS TG model is useful to dissect the individual and combined contributions of HIV and of cocaine administration to the development of CM. Mortality is increased by cocaine administration to TGs compared with WTs. Pathologic changes of fibrosis are found along with echocardiographic features of cardiac dysfunction. It is logical to conclude that cocaine is an important contributor to structural changes, cardiac dysfunction, and mortality in the setting of AIDS.

\section{Methods}

\section{General}

Hemizygous AIDS TG (NL4-3s gag/pol; TG) came from a colony established by Klotman and colleagues (Bruggeman et al, 1997; Kopp et al, 1992) and used by us previously in other studies (Lewis et al, 2000, $2001 b)$. For these $2 \times 2$ studies, both hemizygous TG and $F V B / n(W T)$ mice received either saline or cocaine 

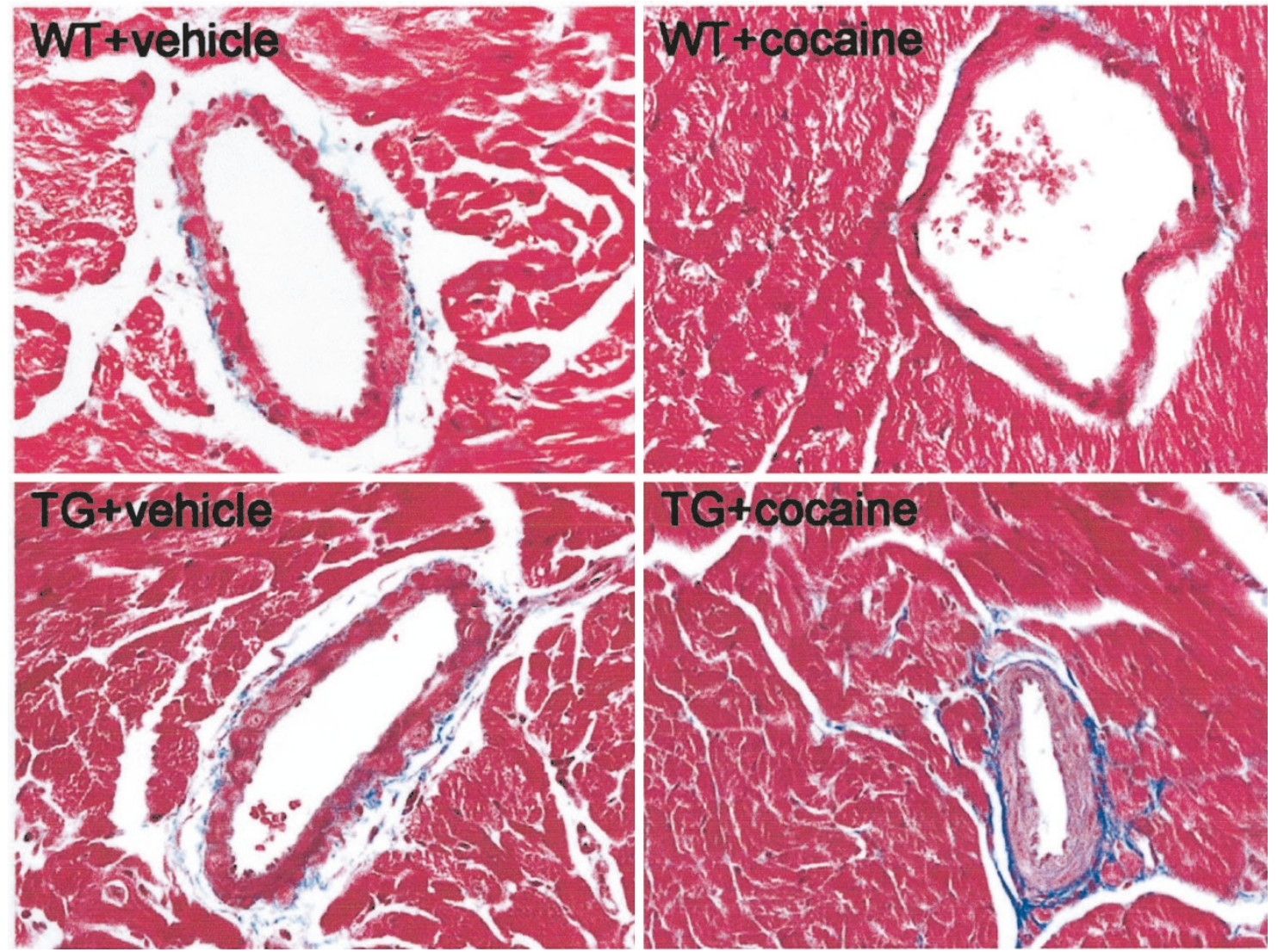

Figure 4.

Histopathologic changes in myocardial sections. Representative sections of myocardium from WT and TG mice treated with saline or cocaine (40 mg/kg/day; 10 days). Perivascular fibrosis is increased in TG + cocaine compared with all other cohorts. Original magnification, $\times 400$; Masson trichrome.

(40 or $80 \mathrm{mg} / \mathrm{kg} /$ day) for either 28 days (chronic) or 10 days (acute). For chronic studies, animals received cocaine via miniosmotic pumps (Alza Corporation, Mountain View, California). For chronic studies, animals were monitored for 28 days. Death was recorded in each cohort by monitoring individually caged animals in the vivarium. Data were analyzed using Kaplan-Meier graph and Graph Pad Prism (San Diego, California). All studies were performed according to institutional guidelines. For acute studies, animals received cocaine via miniosmotic pumps.

\section{Echocardiography in WT and TG Treated with Cocaine}

M-mode echocardiogram studies were performed in 10- to 14-week-old age- and gender-matched (littermate) WT and TGs treated with saline or cocaine (40 $\mathrm{mg} / \mathrm{kg} /$ day; 10 days). Methods are those described previously (Hoit et al, 1997, 1995, 2002) and used by us recently (Lewis et al, 2000, 2001b; Raidel et al, 2002). Briefly, mice were anesthetized with tribromoethanol (0.25 mg/gm of body weight), and 2-dimensionally targeted $\mathrm{M}$-mode studies were performed with a 13-MHz imaging transducer (Acuson Sequoia, Mountain View, California), respectively. M-mode measurements of end-diastolic dimension (EDD), end-systolic dimension (ESD), end-diastolic posterior wall thickness (EDPWTH), and end-diastolic septal wall thickness (EDSWTH) were made from original tracings. Calculated variables included the following: left ventricular fractional shortening $(F S=$ $[E D D-E S D] / E D D)$, and LV mass $(1.06 \times[(E D D+$ EDPWTH + EDSWTH) $\left.\left.]^{3}-(E D D)^{3}\right]\right)$.

\section{ECG in WT and TG Treated with Cocaine}

ECG signals were recorded in conscious mice via AnonyMOUSE ECG Screening Tools (Mouse Specifics, Inc., Boston, Massachusetts). The apparatus includes paw-sized conductive pads embedded in a platform and electronics with solid-state gating programmed to record ECGs when three single pads contact three paws of the unrestrained mouse. The signals were acquired digitally (DI-220; DATAQ Instruments, Inc., Akron, Ohio) at a sampling rate of 2500 samples/second. Data were recorded for 2 to $3 \mathrm{sec}-$ onds to provide a continuous record of 15 to 25 ECG signals.

Data were submitted to the mousespecifics.com Internet site (Mouse Specifics, Inc.) for data analyses by e-MOUSE. e-MOUSE incorporates Fourier analyses and linear time-invariant low pass digital filtering to minimize distortion from common electrical noise and other undesired frequency components. The software uses a peak detection algorithm to find the peak of the R-waves to calculate heart rate. Heart rate 
A.

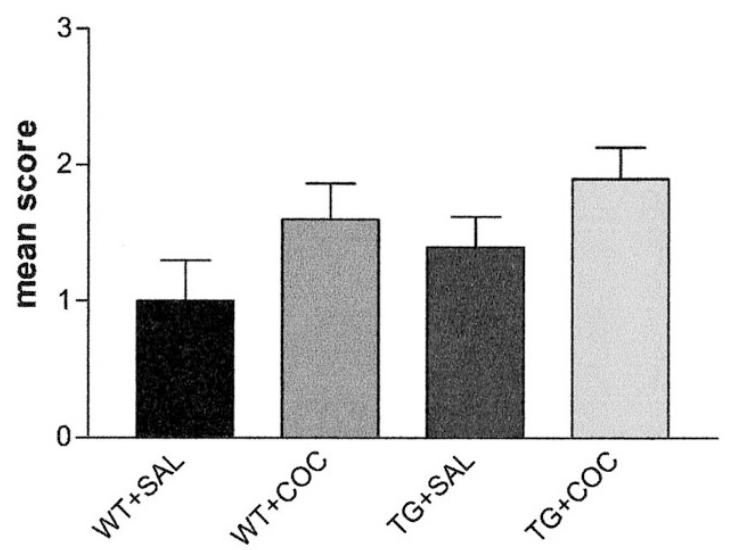

B.

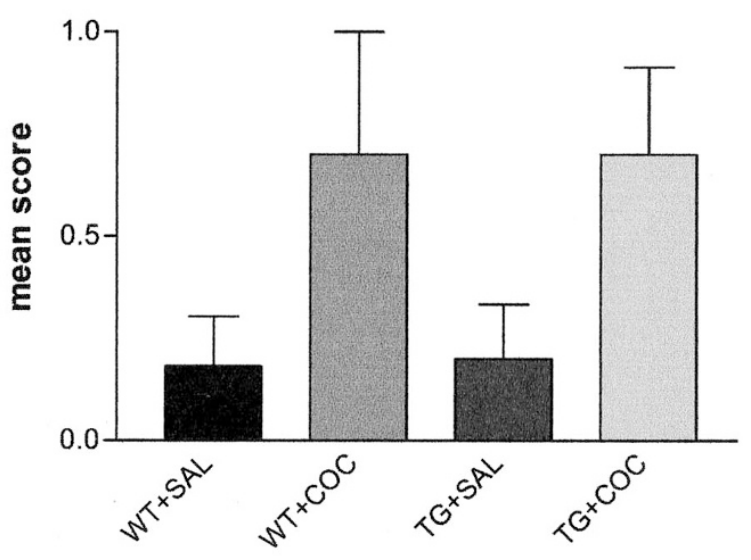

C.

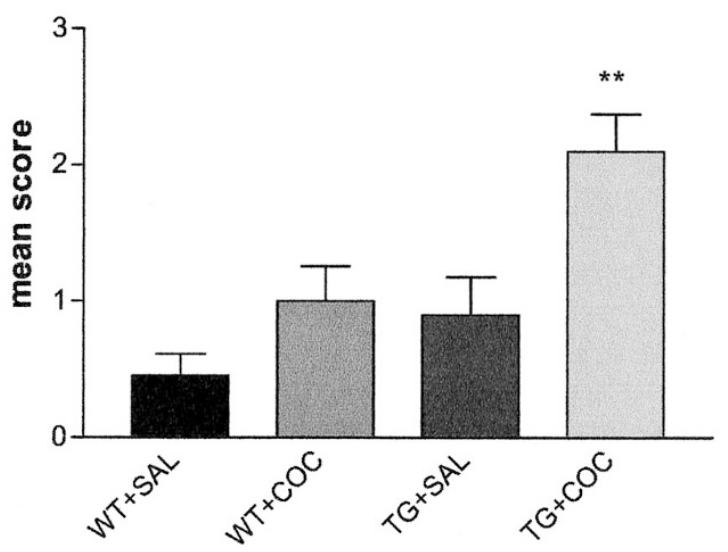

Figure 5.

Histograms of semiquantitative analysis of hearts after treatment of WT and TG with saline or cocaine (40 mg/kg/day; 10 days). Myocyte granularity (A), myocyte fibrosis $(B)$, and perivascular fibrosis $(C)$ were analyzed by blinded review of slides from each of the cohorts. Perivascular fibrosis was significantly increased in the TG + cocaine cohort. $p<0.05$ compared with WT + saline $(n=6-8)$.

variability was calculated as the mean of the differences between sequential heart rates for the complete set of ECG signals. Subsequently, determination of first and second derivatives and algebraic functions searched the ECG signals for probable $P$-wave peaks and onset and termination of QRS complexes. Because the T-wave is not separate from the QRS in rodent ECGs, we defined the T-wave as the first numerical point of inflection occurring after the QRS complex. e-MOUSE calculated the mean of the ECG time intervals for each set of waveforms.

\section{Histologic Sectioning and Evaluation of Hearts in WT and TG Treated with Cocaine}

Histologic correlation with physiologic and pharmacologic data was accomplished in ways that resemble those used by us previously (Lewis et al, 2001b). At the termination of the experiments, myocardial samples were immediately immersion-fixed in neutral buffered formalin. For histopathologic evaluation, each heart slice was dehydrated through a graded series of alcohols and paraffin embedded (uniformly oriented apex down). Serial sections from the paraffinembedded tissue slice $(6 \mu)$ were stained with Masson's trichrome. Two observers blinded to treatment or TG status examined each slide.

A semiquantitative scale was used for histopathologic comparisons within the experiments. Myocyte necrosis was an index of cell death in tissue sections. Total necrotic myocytes were counted in each field examined. Numbers of necrotic cells were measured using the following schema: zero necrotic myocytes per high-power field in ventricular sections was scored as a grade zero; 1 to 3 necrotic myocytes per highpower field was scored as grade $1 ; 4$ to 6 necrotic myocytes per high-power field was scored as grade 2; 7 to 9 necrotic myocytes per high-power field was scored as grade $3 ; 10$ or more necrotic myocytes per high-power field was scored as grade 4. Averages from 10 high-power fields were taken from each slide and numerically scored according to the schema. Upon breaking the code, numerical data obtained from this scoring system were statistically analyzed. Photomicrography of slides was performed with a Nikon photomicroscope (Nikon, Garden City, New York). Interstitial and perivascular fibrosis was evaluated semiquantitatively using the intensity of green counterstain in the interstitium and perivascular space of sections stained with Masson's trichrome method and graded from zero (no fibrosis) to $4+$ (total replacement fibrosis).

\section{References}

Bruggeman LA, Dikman S, Meng C, Quaggin SE, Coffman TM, and Klotman PE (1997). Nephropathy in human immunodeficiency virus-1 transgenic mice is due to renal transgene expression. J Clin Invest 100:84-92.

Calabrese LH, Proffitt MR, Yen-Lieberman B, Hobbs RE, and Ratliff NB (1987). Congestive cardiomyopathy and illness related to the acquired immunodeficiency syndrome (AIDS) associated with isolation of retrovirus from myocardium. Ann Intern Med 107:691-692. 
Georgakopoulos D and Kass D (2001). Minimal forcefrequency modulation of inotropy and relaxation of in situ murine heart. J Physiol 534:535-545.

Grody WW, Cheng L, and Lewis W (1990). Infection of the heart by the human immunodeficiency virus. Am J Cardiol 66:203-206.

Hoit BD, Khan ZU, Pawloski-Dahm CM, and Walsh RA (1997). In vivo determination of left ventricular wall stressshortening relationship in normal mice. Am J Physiol 272: H1047-H1052.

Hoit BD, Khoury SF, Kranias EG, Ball N, and Walsh RA (1995). In vivo echocardiographic detection of enhanced left ventricular function in gene-targeted mice with phospholamban deficiency. Circ Res 77:632-637.

Hoit BD, Kiatchoosakun S, Restivo J, Kirkpatrick D, Olszens K, Shao H, Pao YH, and Nadeau JH (2002). Naturally occurring variation in cardiovascular traits among inbred mouse strains. Genomics 79:679-685.

Inaba T, Stewart DJ, and Kalow W (1978). Metabolism of cocaine in man. Clin Pharmacol Ther 23:547-552.

Isner JM, Estes NA 3rd, Thompson PD, Costanzo-Nordin MR, Subramanian R, Miller G, Katsas G, Sweeney K, and Sturner WQ (1986). Acute cardiac events temporally related to cocaine abuse. N Engl J Med 315:1438-1443.

Karch SB and Billingham ME (1988). The pathology and etiology of cocaine-induced heart disease. Arch Pathol Lab Med 112:225-230.

Klotman PE and Notkins AL (1996). Transgenic models of human immunodeficiency virus type-1. Curr Top Microbiol Immunol 206:197-222.

Kopp JB, Klotman ME, Adler SH, Bruggeman LA, Dickie P, Marinos NJ, Eckhaus M, Bryant JL, Notkins AL, and Klotman PE (1992). Progressive glomerulosclerosis and enhanced renal accumulation of basement membrane components in mice transgenic for human immunodeficiency virus type 1 genes. Proc Natl Acad Sci USA 89:1577-1581.

Lewis W (2000). Cardiomyopathy in AIDS: A pathophysiological perspective. Prog Cardiovasc Dis 43:151-170.

Lewis W (2001a). Absence of cardiac toxicity of zidovudine in infants. N Engl J Med 344:458.

Lewis W (2001b). AIDS cardiomyopathy: Physiological, molecular, and biochemical studies in the transgenic mouse. Ann NY Acad Sci 946:46-56.

Lewis W, Copeland WC, and Day B (2001a). Mitochondrial DNA depletion, oxidative stress and mutation: Mechanisms of nucleoside reverse transcriptase inhibitor toxicity. Lab Invest 81:777-790.

Lewis W, Grupp IL, Grupp G, Hoit B, Morris R, Samarel AM, Bruggeman L, and Klotman P (2000). Cardiac dysfunction occurs in the HIV-1 transgenic mouse treated with zidovudine. Lab Invest 80:187-197.
Lewis W, Haase CP, Raidel SM, Russ RB, Sutliff RL, Hoit BD, and Samarel AM (2001b). Combined antiretroviral therapy causes cardiomyopathy and elevates plasma lactate in transgenic AIDS mice. Lab Invest 81:1527-1536.

Lipshultz SE, Fox CH, Perez-Atayde AR, Sanders SP, Colan SD, McIntosh K, and Winter HS (1990). Identification of human immunodeficiency virus-1 RNA and DNA in the heart of a child with cardiovascular abnormalities and congenital acquired immune deficiency syndrome. Am J Cardiol 66: $246-250$.

Lipshultz SE, Orav EJ, Sanders SP, Hale AR, McIntosh K, and Colan SD (1992). Cardiac structure and function in children with human immunodeficiency virus infection treated with zidovudine [see comments]. N Engl J Med 327:1260-1265.

Raidel SM, Haase C, Jansen NR, Russ RB, Sutliff RL, Velsor LW, Day BJ, Hoit BD, Samarel AM, and Lewis W (2002). Targeted myocardial transgenic expression of HIV Tat causes cardiomyopathy and mitochondrial damage. Am J Physiol Heart Circ Physiol 282:H1672-678.

Ruth JA, Ullman EA, and Collins AC (1988). An analysis of cocaine effects on locomotor activities and heart rate in four inbred mouse strains. Pharmacol Biochem Behav 29:157162.

Shannon RP, Simon MA, Mathier MA, Geng YJ, Mankad S, and Lackner AA (2000). Dilated cardiomyopathy associated with simian AIDS in nonhuman primates. Circulation 101: 185-193.

Shusterman V, Usiene I, Harrigal C, Lee JS, Kubota T, Feldman AM, and London B (2002). Strain-specific patterns of autonomic nervous system activity and heart failure susceptibility in mice. Am J Physiol Heart Circ Physiol 282: H2076-H2083.

Som P, Oster ZH, Wang GJ, Volkow ND, and Sacker DF (1994). Spatial and temporal distribution of cocaine and effects of pharmacological interventions: Wholebody autoradiographic microimaging studies. Life Sci 55:1375-1382.

Twu C, Liu NQ, Popik W, Bukrinsky M, Sayre J, Roberts J, Rania S, Bramhandam V, Roos KP, MacLellan WR, and Fiala M (2002). Cardiomyocytes undergo apoptosis in human immunodeficiency virus cardiomyopathy through mitochondrion- and death receptor-controlled pathways. Proc Natl Acad Sci USA 99:14386-14391.

Volkow ND, Fowler JS, Wolf AP, Wang GJ, Logan J, MacGregor R, Dewey SL, Schlyer D, and Hitzemann R (1992). Distribution and kinetics of carbon-11-cocaine in the human body measured with PET. J Nucl Med 33:521-525.

Wu AY, Forouhar F, Cartun RW, Berman MM, Shiue ST, Louie AT, and Grunnet M (1990). Identification of human immunodeficiency virus in the heart of a patient with acquired immunodeficiency syndrome. Mod Pathol 3:625-630. 